\title{
Disruption of the ventricular myocardial force-frequency relationship after cardiac surgery in children: Noninvasive assessment by means of tissue Doppler imaging
}

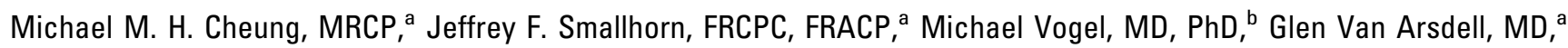
and Andrew N. Redington, MD, FRCPa

From the Division of Cardiology, Hospital for Sick Children, ${ }^{\text {a }}$ Toronto, Ontario, Canada, and Kinderherz-Praxis, ${ }^{\mathrm{b}}$ Munich, Germany.

This study was carried out at the Hospital for Sick Children, Toronto, Ontario, Canada.

Supported by an unrestricted educational grant from GE Vingmed Ultrasound, Horten, Norway, and also by the Heart and Stroke Foundation of Canada. MMHC is supported by a Research Fellowship from the Heart and Stroke Foundation of Ontario.

Received for publication July 4, 2005; revisions received Sept 8, 2005; accepted for publication Sept 15, 2005.

Address for reprints: Andrew Redington, MD, FRCP, Head, Division of Cardiology, The Hospital for Sick Children, 555 University Ave, Toronto, Ontario M5G 1X8, Canada (E-mail: andrew.redington@sickkids.ca).

J Thorac Cardiovasc Surg 2006;131:625-31 $0022-5223 / \$ 32.00$

Copyright $(9) 2006$ by The American Association for Thoracic Surgery

doi:10.1016/j.jtcvs.2005.09.056
Objective: Impaired ventricular function after cardiopulmonary bypass and surgical repair remains a commonly encountered clinical problem. We hypothesized that the well-described impairment of calcium cycling after cardiac surgery would significantly affect the ventricular myocardial force-frequency relationship, which can be measured noninvasively by using the tissue Doppler echocardiography-derived index of contractility isovolumic acceleration.

Methods: Children undergoing repair of congenital heart defects were studied. Rate-related changes in contractility were measured by means of simultaneous atrial pacing and tissue Doppler echocardiography preoperatively and postoperatively.

Results: Although closure of atrial septal defect did not affect ventricular myocardial systolic performance, closure of ventricular septal defect lead to a marked postoperative decrease of basal contractile force $\left(2.0 \pm 0.7 \mathrm{~m} / \mathrm{s}^{2}\right.$ preoperatively vs $1.0 \pm 0.7 \mathrm{~m} / \mathrm{s}^{2}$ postoperatively, $\left.P<.02\right)$. Furthermore, the force-frequency relationship curves were significantly different $(P<.001)$, with a reduced force-rate trajectory, and also peak force was attained. Neonates undergoing the arterial switch procedure showed the most marked postoperative decrease of isovolumic acceleration at basal heart rates and force-frequency relationship with reduced trajectory and peak force development $(P<.0001)$.

Conclusions: This is the first clinical study describing the noninvasive acquisition of ventricular force-frequency relationships in children undergoing operations for congenital heart disease. There is a marked variability in response, ranging from no effect in patients undergoing atrial septal defect closure to a profound reduction in myocardial contractile responses after neonatal arterial switch. This simple noninvasive method allows measurement of a hitherto rarely examined property of the myocardium, an understanding of which might allow refinement of myocardial protection and postoperative myocardial support.

$\mathrm{M}$ ost evidence points toward rate-related changes in calcium cycling within the cardiomyocyte as the underlying subcellular mechanism for the forcefrequency relationship (FFR) or treppe effect. ${ }^{1,2}$ First described by Bowditch, ${ }^{3}$ this intrinsic property of cardiac muscle to alter contractile force with changes in heart rate has been shown in in vitro experiments ${ }^{4}$ and invasive clinical studies to be impaired in disease states, such as dilated and ischemic cardiomyopathy ${ }^{5,6}$ and ventricular hypertrophy. ${ }^{7}$ We have recently shown that it is possible to measure the FFR of the left and right ventricle in an animal model by using the novel tissue Doppler-derived index of contractility, isovolumic acceleration (IVA). ${ }^{8,9}$ This noninvasive method can be easily applied in the clinical situation and should allow new insights into human myocardial disease. 


\author{
Abbreviations and Acronyms \\ ASD $=$ atrial septal defect \\ FFR $=$ force-frequency relationship \\ IVA $=$ isovolumic acceleration \\ TDI $=$ tissue Doppler imaging \\ TGA $=$ transposition of the great arteries \\ TOF $=$ tetralogy of Fallot
}

Impaired ventricular function after cardiopulmonary bypass and surgical repair remains a commonly encountered clinical problem. We hypothesized that the well-described impairment of calcium cycling after cardiopulmonary bypass $^{10}$ would significantly affect the measured ventricular myocardial FFR. The ability to measure this physiologic property in children undergoing cardiac surgery would not only increase our knowledge of postoperative changes in myocardial contractility but also potentially guide modification of ventricular function by therapeutic changes in heart rate and inotropic support. The aim of this initial study was to examine the changes in the FFR in the early postoperative period in selected groups of children undergoing $\mathrm{CPB}$ and surgical repair of congenital heart defects.

\section{Methods \\ Patients}

The study protocol was approved by the institution's research ethics board. Informed consent was obtained before enrollment in the study. All subjects were studied after achievement of general anesthesia at the time of surgical repair. Cardiopulmonary bypass techniques with blood cardioplegia were standardized for all patients. Muscle relaxants were used in all patients, with maintenance of anesthesia obtained by using a combination of intravenous fentanyl and inhaled isoflurane. Patients undergoing Fontan completion after a previous Glenn shunt, neonates undergoing the arterial switch procedure for transposition of the great arteries (TGA), and patients requiring surgical repair of atrial septal defects (ASDs), ventricular septal defects (VSDs), or tetralogy of Fallot (TOF) were studied. This population thus provided patients with a range of ages and also varying durations of bypass and crossclamp times. The degree of inotropic support was quantified by using a scoring system calculated as the sum of all inotrope doses, correcting for potency. ${ }^{11,12}$

\section{Echocardiography}

Our method has been previously described. ${ }^{8,9}$ Briefly, tissue Doppler imaging (TDI) was performed by using a System V ultrasound scanner (GE Vingmed) with a $5-\mathrm{MHz}$ or $10-\mathrm{MHz}$ probe. The heart was imaged from a transthoracic apical 4-chamber view preoperatively and postoperatively, and imaging parameters were optimized to yield the highest frame rate possible $(>165$ frames per second). Color-coded myocardial velocities were recorded from the basal midwall immediately below $(0.5 \mathrm{~cm})$ the insertion of the mural leaflet of the atrioventricular valve of the dominant ventricle in those patients with univentricular hearts and of the left ventricle in those patients with biventricular hearts. Recordings were made simultaneously with electrocardiography. A cineloop of at least 3 consecutive cardiac cycles coinciding with end expiration was stored digitally for offline analysis.

\section{Pacing Protocol}

Preoperatively, patients were studied in the operating room after induction of anesthesia and insertion of monitoring lines. Subjects were atrially paced through the transesophageal route with a $5 \mathrm{~F}$ bipolar catheter (TAPCATH 205, CardioCommand, Inc) connected to a high-output pulse generator (model 2380, Medtronic). The optimum depth of insertion was judged from the size of the atrial signal. ${ }^{13}$ Echocardiographic measurements were made at the resting nonpaced heart rate and during atrial pacing from the resting heart rate plus 5 beats/min up to a maximum of 200 beats/min in increments of 10 beats/min. Studies were stopped if systemic blood pressure decreased by $30 \%$ of baseline or atrioventricular block occurred.

Postoperatively, patients were studied at 3, 6, 12, and 24 hours, provided the patients remained intubated, sedated, and ventilated. Pacing was through the epicardial route using the temporary right atrial pacing wires placed routinely at the time of operation and connected to an external pulse generator (model 5388, Medtronic). The pacing protocol was identical to that used preoperatively.

\section{Blood Sampling}

Arterial blood samples were taken coincident with echocardiographic assessments and analyzed for acid-base status, levels of lactate, and ionized calcium.

\section{Data Analysis}

Echopac software (GE Vingmed) was used to analyze the stored TDI data by using our previously described method. ${ }^{8,9}$ The resulting Doppler spectrum was displayed and analyzed by using commercially available software (Echopac, GE Vingmed). Systolic ejection (s velocity), as well as IVA, were measured with electronic calipers. Acceleration was calculated as the difference between baseline and peak velocity divided by the time interval. We have previously published interobserver and intraobserver variability data for the measurement of IVA. There was a $9.8 \% \pm$ $7.8 \%$ interobserver and $5.7 \% \pm 4.6 \%$ intraobserver variability of measurement of IVA, with satisfactory Bland-Altman analysis of interobserver variability. ${ }^{9}$ The maximal percentage change in IVA from the preoperative study after cardiopulmonary bypass was taken at the lowest comparable postoperative heart rate with the lowest value of IVA.

\section{Statistical Analysis}

Data are expressed as means \pm standard deviation. In the figures data are displayed as means \pm standard error of the mean. Age at the time of the operation, bypass times, time to extubation, and inotropic scores were analyzed by means of 1-way analysis of variance with post-hoc Bonferroni comparison. TDI-derived FFR curves at 2 different time intervals within a patient group were compared by means of 2-way analysis of variance with post-hoc Bonferroni comparison. Those with more than 2 curves were analyzed by means of 1-way analysis of variance with post-hoc Bonferroni comparison. Correlation was performed with the Pear- 
TABLE 1. Mean \pm SD age, bypass and crossclamp durations, time to extubation, and inotrope scores at 3 hours postoperatively for each diagnostic group

\begin{tabular}{|c|c|c|c|c|c|}
\hline & $\operatorname{ASD}(n=4)$ & VSD $(n=11)$ & TOF $(n=5)$ & Fontan $(n=10)$ & TGA $(\mathbf{n}=11)$ \\
\hline Age (y) & $3.4 \pm 1.2$ & $2.0 \pm 2.5$ & $0.37 \pm 0.1$ & $4.3 \pm 1.5$ & $0.03 \pm 0.02^{*}$ \\
\hline Bypass duration (min) & $35 \pm 9$ & $82 \pm 26$ & $98 \pm 23$ & $80 \pm 49$ & $105 \pm 31 \dagger$ \\
\hline Crossclamp duration (min) & $16 \pm 17 \ddagger$ & $55 \pm 14$ & $62 \pm 16$ & $9 \pm 20 \ddagger$ & $61 \pm 26$ \\
\hline Time to extubation $(\mathrm{h})$ & $5.3 \pm 2.3$ & $12.5 \pm 10.4$ & $22.6 \pm 6.7$ & $13.1 \pm 12.9$ & $52.2 \pm 20 \S$ \\
\hline Inotrope score at $3 \mathrm{~h}$ postop $\left(\mu \mathrm{g} \cdot \mathrm{kg}^{-1} \cdot \min ^{-1}\right)$ & $0 \pm 0$ & $8.8 \pm 3.0 \uparrow$ & $11.8 \pm 3.2$ & $13.2 \pm 3.9$ & $15.3 \pm 0.9 \|$ \\
\hline
\end{tabular}

$A S D$, Atrial septal defect; $V S D$, ventricular septal defect; TOF, tetralogy of Fallot; TGA, transposition of the great arteries. $* P<.05$ versus all other patient groups. $\dagger P<.05$ versus ASD group but no difference versus any other patient group. $¥$ No difference between ASD and Fontan groups but significantly shorter than the other 3 groups $(P<.05)$. §Significantly longer time to extubation $(P<.001$ vs ASD, VSD, and Fontan groups and $P<.01$ vs TOF group). $\|$ Significantly greater than scores for ASD and VSD groups $(P<.001$ for both) and also TOF group $(P<.05)$. q[Significantly lower than Fontan group.

son method. Data were analyzed with Graphpad Prism (GraphPad Software, Inc).

\section{Results}

Patients

All 11 neonates (age, $10 \pm 6$ days) with TGA had the usual coronary pattern, and only one had a small VSD, which was closed during surgical intervention. All 11 underwent uncomplicated arterial switch procedures. There were 4 patients with ASDs (age, $3.4 \pm 1.2$ years) that were closed either by means of direct suture or patch closure. The 11 patients with VSDs (age, $2.0 \pm 2.5$ years) underwent repairs by means of patch closure through a transatrial approach. There were 5 patients with TOF (age, $0.4 \pm 0.1$ years), all of whom underwent repair by means of a transatrial transpulmonary approach. The 10 patients with functionally single ventricles (age, $4.5 \pm 1.5$ years) all underwent Fontan completion with a fenestrated extracardiac conduit with $(\mathrm{n}=3)$ or without $(\mathrm{n}=7)$ aortic crossclamps. As expected, the TGA group was significantly younger than all the other groups studied. Comparison of age between the other 4 groups showed no significant difference.

Age, bypass and aortic crossclamp times, inotrope scores at 3 hours postoperatively, and time to extubation are shown in Table 1. Only inotrope scores at 3 hours postoperatively are shown for comparison because in some groups patients were extubated and weaned from inotropic support early in the postoperative period. The mean inotropic score for the TGA group $(15.3 \pm 0.9)$ was significantly greater than for the $\operatorname{ASD}(0 \pm 0, P<.001)$, VSD $(8.8 \pm 3.0, P<.001)$ and TOF $(11.8 \pm 3.2, P<.05)$ groups. There was no significant difference between the TGA and Fontan groups (13.2 \pm 3.9 , $P>.05)$ or between the TOF and Fontan groups $(P>.05)$. The VSD group had significantly lower inotrope scores than the Fontan group $(P<.01)$. Mean bypass times for the patients with TGA were significantly longer than those of the patients with ASDs $(P<.05)$, but there was no difference in comparison with any other group. Although there was no significant difference between the aortic crossclamp durations for patients with ASDs and those undergoing the
Fontan procedure, these were significantly shorter than in the other 3 groups $(P<.05)$. There was no significant difference among these latter 3 groups.

No patients were receiving inotropic infusions preoperatively, and none of the patients with ASDs received inotropic drugs postoperatively.

Time to extubation was significantly longer in the TGA group in comparison with that seen in all other groups $(P<$ .001 vs the ASD, VSD, and Fontan groups and $P<.01$ vs the TOF group). There was no significant difference among the other 4 groups.

At the time of each study, arterial blood samples showed $\mathrm{pH}$ and levels of ionized calcium to be within the normal ranges. There were no differences in inotrope scores or time to extubation in those patients undergoing Fontan completion with or without aortic crossclamping.

\section{Force-frequency Relationships}

The measurements of changes in IVA with pacing-induced tachycardia for each patient group are shown in Figures 1 through 5. For the patients undergoing ASD repair (Figure 1)

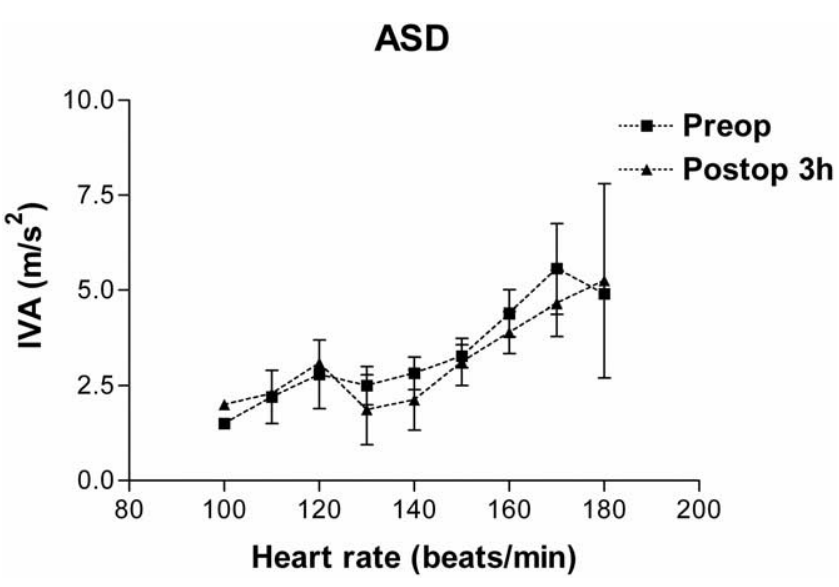

Figure 1. Pacing-induced changes in isovolumic acceleration (IVA) for patients undergoing surgical repair of atrial septal defect (ASD). Error bars represent standard error of the mean. 


\section{Fontan completion}

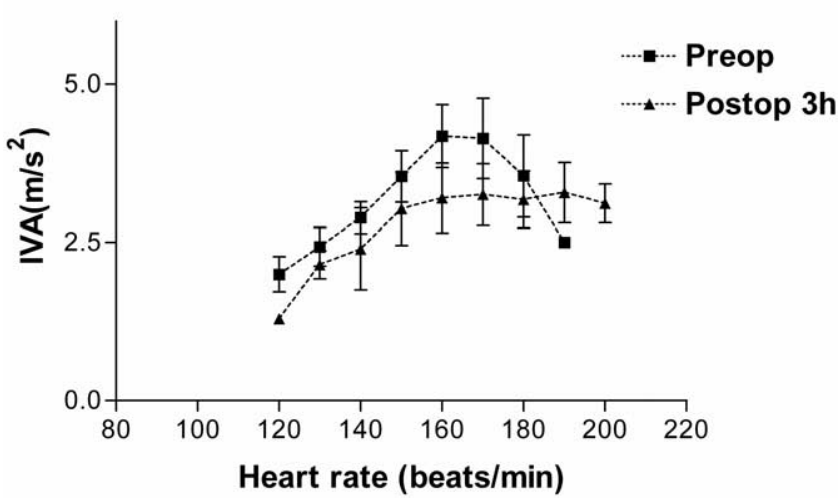

Figure 2. Pacing-induced changes in isovolumic acceleration (IVA) for patients undergoing Fontan completion. Error bars represent standard error of the mean.

and Fontan completion (Figure 2), only preoperative and 3-hour postoperative measurements are shown because there were so few patients with later postoperative measurements. There were no significant differences between measurements of preoperative and postoperative FFR within each of these groups. There was no difference in the postoperative FFRs in patients in the Fontan group undergoing repair with and without aortic crossclamping.

Comparison of the preoperative and 3-hour postoperative data for the VSD group (Figure 3), however, shows there to be a marked decrease of basal (heart rate of 130 beats $/ \mathrm{min}$ ) contractility $\left(2.0 \pm 0.7 \mathrm{~m} / \mathrm{s}^{2}\right.$ preoperatively vs $1.0 \pm 0.7 \mathrm{~m} / \mathrm{s}^{2}$ postoperatively, $P<.02)$. Furthermore, the FFR curves are significantly different $(P<.0001)$, with a reduced force-rate trajectory, and also peak contractility was attained.

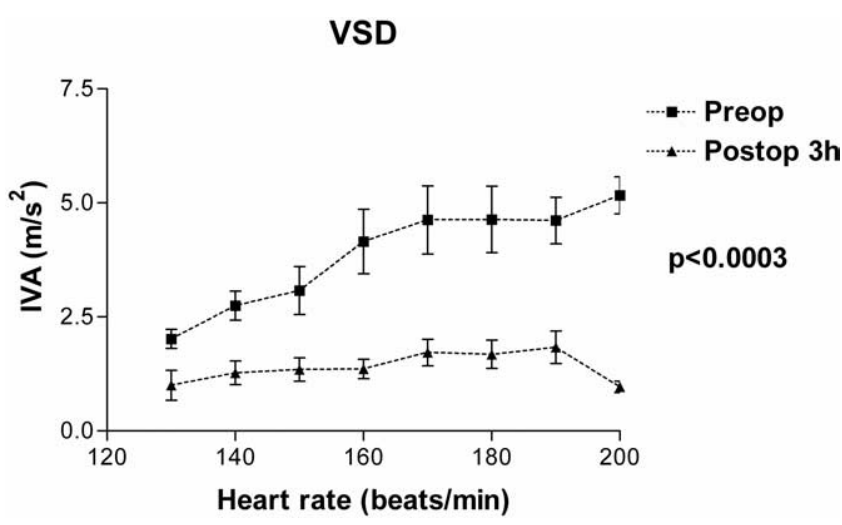

Figure 3. Pacing-induced changes in isovolumic acceleration (IVA) for patients undergoing surgical repair of ventricular septal defect (VSD). Error bars represent standard error of the mean.

\section{Tetralogy of Fallot}

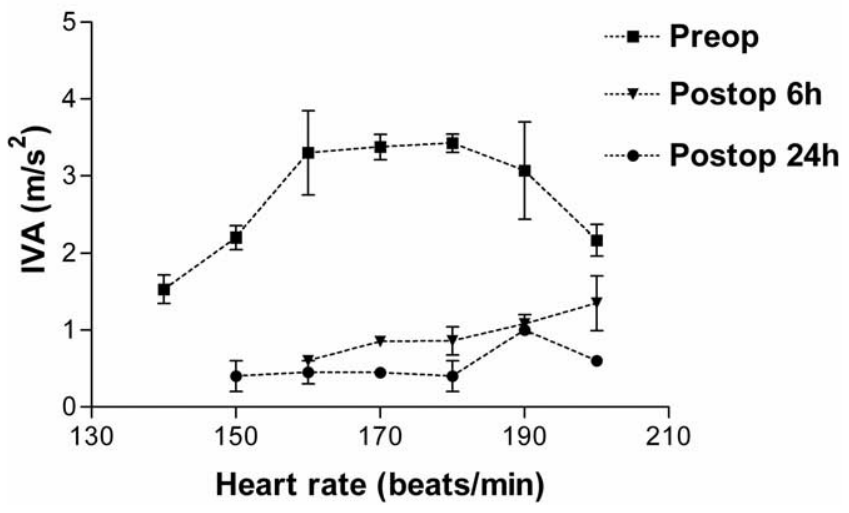

Figure 4. Pacing-induced changes in isovolumic acceleration (IVA) for patients undergoing surgical repair of tetralogy of Fallot. Error bars represent standard error of the mean.

Data from the TOF group (Figure 4) similarly show a marked decrease of basal contractile force and also a profound change in the FFR. It is difficult, however, to compare these changes statistically because all of the patients in this group were relatively tachycardic compared with preoperative assessment.

The TGA group data (Figure 5) show the most marked decrease of IVA at basal heart rates, with reduced trajectory and development of peak contractility postoperatively. The postoperative FFR curves were significantly different from the preoperative assessment $(P<.001$ for preoperative values vs both 6 and 24 hours postoperatively, 1-way analysis of variance with post-hoc Bonferroni comparison). Although there was some improvement in peak contractility

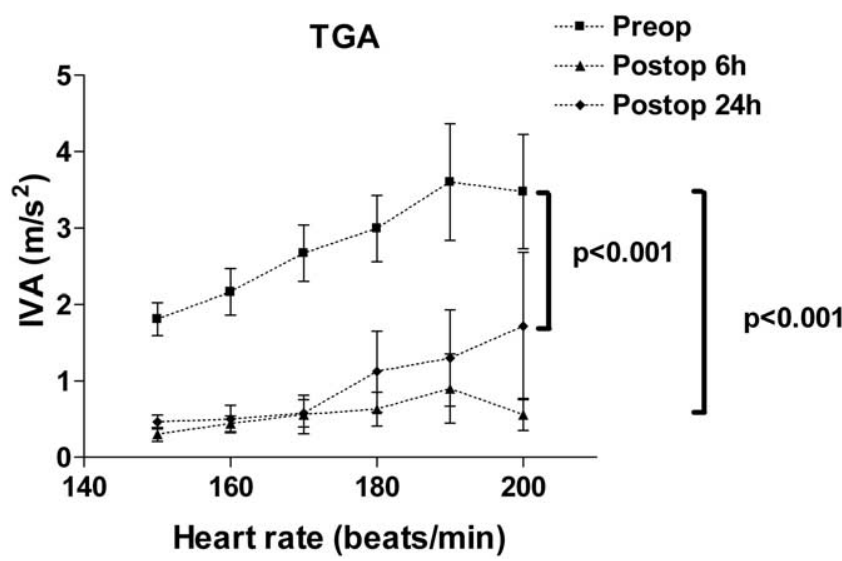

Figure 5. Pacing-induced changes in isovolumic acceleration (IVA) for patients with transposition of the great arteries (TGA) undergoing the arterial switch procedure. Error bars represent standard error of the mean. 


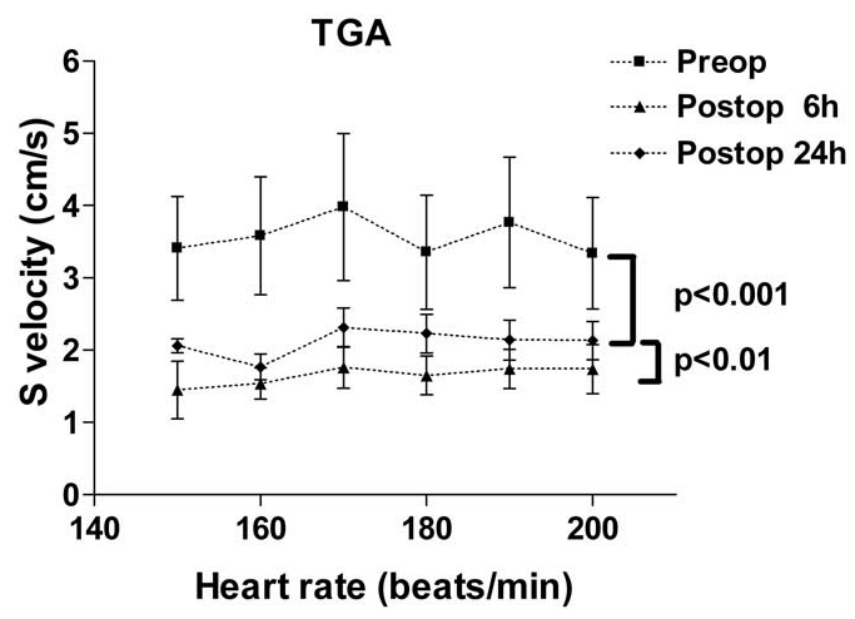

Figure 6. Pacing-induced changes in s velocity for patients with transposition of the great arteries (TGA). Error bars represent standard error of the mean.

developed at 24 hours compared with 6 hours postoperatively, this was not statistically significant $(P>.05)$. There were no significant changes in inotropic scores at $3,6,12$, and 24 hours postoperatively $(15.3 \pm 0.9,12.9 \pm 4.1,16.4 \pm$ 5.4 , and $12.9 \pm 3.9$, respectively; $P=.20$ ).

\section{S Velocity}

Data for s velocity of the TGA group are shown for comparison (Figure 6). It was not possible to demonstrate the myocardial FFR with measurements of s velocity. There were, however, significant differences in the $s$ velocity measurements at the time intervals displayed. Preoperative values and those at 24 hours postoperatively were significantly greater than at 6 hours postoperatively $(P<.001$ and $P<.01$, respectively).

\section{Percentage Change in IVA}

The maximal percentage change in IVA was compared with the duration of cardiopulmonary bypass and also the duration of aortic crossclamping. There was no significant correlation between change in IVA and the duration of bypass ( $r=-0.05, P=.8$ ). There was, however, a weak but significant correlation between percentage change in IVA and the duration of aortic crossclamping $(r=-0.3, P<$ .05 , Figure 7).

\section{Discussion}

For the first time, using the noninvasively determined tissue Doppler-derived index of contractility IVA, we have demonstrated the feasibility of studying the FFR in children undergoing cardiac surgery. This study shows the deleterious effect of the process of cardiac surgery on the human ventricular myocardial FFR in vivo, with decreases of both

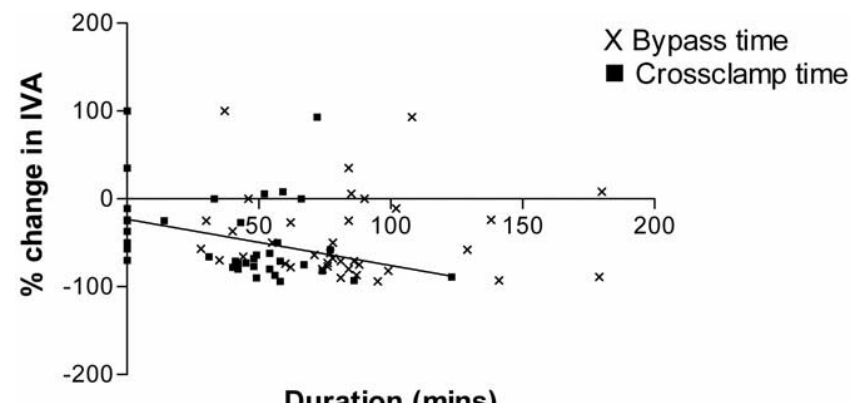

Figure 7. Comparison of maximal percentage change in isovolumic acceleration (IVA) with duration of cardiopulmonary bypass and aortic crossclamp time. The best-fit line for comparison with aortic crossclamp duration is shown $(r=-0.3, P<.05)$.

basal and also rate-related changes in contractility. The degree of impairment of this intrinsic myocardial property is variable between and within the anatomic subgroups and underscores the importance of assessing heart rate-related changes in postoperative myocardial function.

\section{Postoperative Basal Contractile Function}

Although the detrimental effects of ischemia and reperfusion on ventricular performance are well described, ${ }^{10}$ clinical assessment of postoperative contractile function is complicated by significantly altered loading conditions. ${ }^{14}$ Analysis of ventricular pressure-volume data remains the gold standard for assessment; however, this is an invasive technique requiring specialized equipment and is impractical in the intensive care unit setting. ${ }^{15} \mathrm{We}$ have recently validated the novel tissue Doppler-derived index, IVA, as a measure of ventricular contractile function. IVA is sensitive to contractile change and relatively independent of changes in loading conditions $^{8,9}$ over a physiologic range that might be expected postoperatively. It can also describe the myocardial FFR and therefore is potentially ideal for describing postoperative changes in function related to crossclamping and CPB. Indeed, most patients experienced an increase in heart rate after surgical intervention, and therefore we chose to express changes in basal contractile function as changes in IVA at the basal heart rate postoperatively compared with IVA measured at that heart rate, with atrial pacing measured preoperatively.

Patients after ASD repair showed no significant difference in basal contractile function, suggesting little effect of the effect of aortic crossclamping and cardioplegia combined with a short period of CPB. It is important to note that the patients with ASDs were not receiving inotropic agents. All patients in the other groups were receiving inotropes when evaluated. Thus the lack of any change in basal IVA in the Fontan group is difficult to interpret because the 
inotropic therapy score for this group was relatively high, perhaps normalizing myocardial dysfunction induced by surgical repair. ${ }^{16}$ Nonetheless, it is likely that any degree of dysfunction was less than in the TGA and TOF groups, in whom inotropic therapy scores were not significantly different and reduction in basal IVA was significantly greater. Because the degree of myocardial injury after ischemiareperfusion injury is related to the duration of ischemia, ${ }^{10}$ we postulate that this observation is in part due to the shorter aortic crossclamp and bypass durations for this patient group. Indeed, there was a weak but significant correlation between percentage change in IVA after surgical intervention and duration of aortic crossclamping. The responses of individual patients to the process of cardiac surgery will invariably be somewhat idiosyncratic, however.

\section{Intergroup Differences in Perioperative FFR}

There were also differences between the patient groups studied in terms of relative change in the FFR. There were no significant changes in the FFR curves postoperatively in either the Fontan or the ASD groups, whereas there were marked changes in all other groups. The same considerations regarding the effect of inotropic support or lack of it pertain to the interpretation of changes in FFR. However, it is quite clear that patients after VSD closure, repair of TOF, and, in particular, after the arterial switch procedure have significantly greater myocardial dysfunction.

We studied patients with different diagnoses that are routinely repaired at different ages and are associated with varying degrees of preoperative shunting and cyanosis. The effect of preoperative cyanosis on the myocardial response to ischemia and reperfusion is uncertain. We therefore included patients with VSDs to provide a comparative group for those with TOF. The degree of postoperative disruption to the FFR was comparable between these 2 groups, demonstrating minimal effect of preoperative cyanosis.

The transposition group showed differences in heart raterelated changes in IVA and s velocity. As in our validative studies, the ejection-phase index, s velocity, did not change significantly with increasing heart rate. This is presumably due to the greater dependence of this ejection-phase index on preload $^{17}$ and its inability to track the FFR. In the absence of any significant change in inotrope score, the 3 curves (Figure 6) did show a significant progression, with a nadir at 6 hours and a significant degree of recovery at 24 hours postoperatively, which mirrors the changes in cardiac index described by Wernovsky and colleagues. ${ }^{11}$ These changes in s velocity are difficult to interpret in the absence of other hemodynamic data, but the lack of change in IVA would indicate that this progression is not due to an improvement in intrinsic contractility. Although speculative, we would suggest the changes in systemic vascular resistance described by Wernovsky and colleagues might ex- plain these observed changes in $\mathrm{s}$ velocity because this ejection-phase index is known to be affected by changes in afterload.

We hypothesize therefore that the improvement in cardiac index described by Wernovsky and colleagues ${ }^{11}$ appears not to be based on improved contractility but rather is due to the favorable change in afterload.

\section{Mechanism of Dysfunction}

Analysis of FFRs allows insights into the cellular basis of myocardial dysfunction. The FFR is a manifestation of calcium cycling within the cardiomyocyte, as demonstrated by in vitro studies with calcium-sensitive dyes. ${ }^{18}$ The function of specific proteins involved in calcium cycling, such as the adenosine triphosphate-sensitive sarcoendoplasmic reticulum calcium channel, have been shown to be decreased after cardiopulmonary bypass. ${ }^{19}$ This protein is intimately involved in the process of excitation-contraction coupling. The effect of increasing stimulation rate is thought to be an increase in activity of sarcoplasmic reticulum $\mathrm{Ca}^{2+}$-ATPase (SERCA) through phosphorylation by calcium-dependent calmodulin kinase. ${ }^{20}$ The rate-dependent intracellular flux of calcium can be demonstrated through the use of calcium dyes, such as aequorin. ${ }^{1}$ Therefore, as we hypothesized, we demonstrated impairment of the ventricular myocardial FFR after cardiopulmonary bypass that appears to correlate with the degree of injury induced by ischemia and reperfusion rather than cardiopulmonary bypass-induced effects.

\section{Study Limitations}

The duration of cardiopulmonary bypass and crossclamping was variable within and between groups. Despite this inhomogeneity, however, we were able to demonstrate consistent responses for each of these patient groups.

Inherent to any study of postsurgical responses in patients with different diagnoses is the difference in age at the time of repair. To examine the possible effect of age on perioperative change in the myocardial FFR, because all of the patients with TGA underwent repair in the neonatal period, we included patients with TOF as a comparison group. These were relatively older children but with a comparable degree of cyanosis. At least in the early postoperative period, these 2 groups showed a similar pattern of perioperative myocardial response. Indeed, contractile dysfunction persists at 24 hours postoperatively in both groups. These 2 groups of patients are clearly not directly comparable because most of the postnatal changes in myocardial function are complete by 2 months of age. In a study of this nature, however, it is difficult to make direct comparisons of the effect of age on any change in FFR, and this factor should be considered in interpreting these data.

The repair of lesions with different levels of intracardiac shunting has contrasting implications for ventricular geom- 
etry and loading conditions. Assessment of ventricular function postoperatively therefore needs to account for these changes when comparing different patient groups. This factor further emphasizes the need for indices, such as IVA, which is relatively independent of changes in loading conditions within a physiologic range. Nonetheless, the responses of contractile indices, including IVA, are difficult to validate in human subjects, in which a complex interplay of changes in loading conditions and myocardial contractility are often interrelated. However, in our previous study we have shown IVA and $\mathrm{dP} / \mathrm{dt}_{\max }$ to react similarly in an experimental analysis of the FFR, ${ }^{9}$ and we have also shown the percentage change in IVA to correlate significantly with the percentage change in end-systolic elastance in human subjects after congenital cardiac surgery. ${ }^{21}$

Although there is strong evidence to support myocyte calcium cycling as being the underlying mechanism for the myocardial FFR and its abnormalities, we have not shown concurrent changes in the rate of calcium cycling after cardiopulmonary bypass in this study. There are currently, however, no easily applicable techniques to measure acute changes in calcium flux in vivo clinically.

\section{Conclusions}

For the first time, we have demonstrated in vivo the perioperative changes in the ventricular myocardial FFR. The novel tissue Doppler-derived index, IVA, can be used at the bedside to examine contractility. The technique of assessment of the myocardial contractile response to pacing provides a simple noninvasive method to measure a hitherto rarely examined property of the myocardium in vivo.

\section{References}

1. Gwathmey JK, Slawsky MT, Perreault CL, Briggs GM, Morgan JP, Wei JY. Effect of exercise conditioning on excitation-contraction coupling in aged rats. J Appl Physiol. 1990;69:1366-71.

2. Alpert NR, Leavitt BJ, Ittleman FP, Hasenfuss G, Pieske B, Mulieri LA. A mechanistic analysis of the force-frequency relation in nonfailing and progressively failing human myocardium. Basic Res Cardiol. 1998;93(suppl 1):23-32.

3. Bowditch H. Uber die Eigentumlichkeiten der Reizbarkeit, welche die Muskelfasern des Herzens zeigen. Ber Konigl Sachs Ges Wissen. 1871; 23:652.

4. Anderson PA, Manring A, Serwer GA, Benson DW, Edwards SB, Armstrong BE, et al. The force-interval relationship of the left ventricle. Circulation. 1979;60:334-48.

5. Morgan JP, Erny RE, Allen PD, Grossman W, Gwathmey JK. Abnormal intracellular calcium handling, a major cause of systolic and diastolic dysfunction in ventricular myocardium from patients with heart failure. Circulation. 1990;81(suppl III):III21-32.
6. Pieske B, Maier LS, Bers DM, Hasenfuss G. Ca2+ handling and sarcoplasmic reticulum $\mathrm{Ca} 2+$ content in isolated failing and nonfailing human myocardium. Circ Res. 1999;85:38-46.

7. Liu CP, Ting CT, Lawrence W, Maughan WL, Chang MS, Kass DA. Diminished contractile response to increased heart rate in intact human left ventricular hypertrophy. Systolic versus diastolic determinants. Circulation. 1993;88:1893-906.

8. Vogel M, Schmidt MR, Kristiansen SB, Cheung M, White PA, Sorensen K, et al. Validation of myocardial acceleration during isovolumic contraction as a novel noninvasive index of right ventricular contractility: comparison with ventricular pressure-volume relations in an animal model. Circulation. 2002;105:1693-9.

9. Vogel M, Cheung MM, Li J, Kristiansen SB, Schmidt MR, White PA, et al. Noninvasive assessment of left ventricular force-frequency relationships using tissue Doppler-derived isovolumic acceleration: validation in an animal model. Circulation. 2003;107:1647-52.

10. Kloner RA, Jennings RB. Consequences of brief ischemia: stunning, preconditioning, and their clinical implications: part 1. Circulation. 2001;104:2981-9.

11. Wernovsky G, Wypij D, Jonas RA, Mayer JE Jr, Hanley FL, Hickey $\mathrm{PR}$, et al. Postoperative course and hemodynamic profile after the arterial switch operation in neonates and infants. A comparison of low-flow cardiopulmonary bypass and circulatory arrest. Circulation. 1995;92:2226-35.

12. Shore S, Nelson DP, Pearl JM, Manning PB, Wong H, Shanley TP, et al. Usefulness of corticosteroid therapy in decreasing epinephrine requirements in critically ill infants with congenital heart disease. Am J Cardiol. 2001;88:591-4.

13. Benson DW Jr, Sanford M, Dunnigan A, Benditt DG. Transesophageal atrial pacing threshold: role of interelectrode spacing, pulse width and catheter insertion depth. Am J Cardiol. 1984;53:63-7.

14. Anderson PAW. Developmental cardiac physiology and myocardial function. In: Hoffman JIE, editor. Pediatric cardiovascular medicine. New York: Churchill Livingstone; 2000. p. 35-59.

15. Kass DA, Yamazaki T, Burkhoff D, Maughan WL, Sagawa K. Determination of left ventricular end-systolic pressure-volume relationships by the conductance (volume) catheter technique. Circulation. 1986; 73:586-95.

16. Kambayashi M, Miura T, Oh BH, Rockman HA, Murata K, Ross J Jr. Enhancement of the force-frequency effect on myocardial contractility by adrenergic stimulation in conscious dogs. Circulation. 1992;86: $572-80$.

17. Brodin L, Lind B, Lang H, Kallner G, Soderqvist E, van der Linden J, et al. The influence of changes in loading conditions on myocardial velocities measured by tissue Doppler [abstract]. Circulation. 2000; 102:383-4.

18. Alpert NR, Leavitt BJ, Ittleman FP, Hasenfuss G, Pieske B, Mulieri LA. A mechanistic analysis of the force-frequency relation in nonfailing and progressively failing human myocardium. Basic Res Cardiol. 1998;93:23-32.

19. Flesch M, Maack C, Cremers B, Baumer AT, Sudkamp M, Bohm M. Effect of beta-blockers on free radical-induced cardiac contractile dysfunction. Circulation. 1999;100:346-53.

20. Schouten VJ. Interval dependence of force and twitch duration in rat heart explained by $\mathrm{Ca} 2+$ pump inactivation in sarcoplasmic reticulum. J Physiol. 1990;431:427-44.

21. Vogel M, Derrick G, White PA, Cullen S, Aichner H, Deanfield J, et al. Systemic ventricular function in patients with transposition of the great arteries after atrial repair: a tissue Doppler and conductance catheter study. J Am Coll Cardiol. 2004;43:100-6. 